andern zu bestehen; beiden gehört das Verdienst, für unsere Zeit das geographische Antlitz der Schweiz in Wort und Bild umfassend festgehalten zu haben.

Das Buch ist flüssig und klar geschrieben. Das war gewiß keine Kleinigkeit, galt es doch, dreißigmal nacheinander einen im Grunde genommen ähnlichen Stoff sprachlich zu meistern. Zwar finde ich nicht alle sprachlichen Formulierungen glücklich, und auch nicht alle Ausdrücke, so z.B. nicht die zu oft wiederkehrende «Talung» für das schöne und einfache Wort Tal, oder die Tributäre für die $\mathrm{Zu}$ - und Nebenflüsse. Dem Band sind zahlreiche, bestens ausgeführte Strichzeichnungen beigegeben, die den Text in trefflicher Weise unterstützen.

\title{
SELINUNTE, TIPO DI PAESAGGIO STORICO SICILIANO
}

\author{
LAURENT BRIDEL
}

Fra le numerose reliquie culturali dell'antica Sicilia, Selinunte occupa uno dei posti più notevoli.

Fu una delle città fondate dai Greci a partire dell'VIII secolo a. C. Se sappiamo all'incirca quali furono le principali produzioni della Sicilia a quell'epoca - olio, cereali, vino, montoni, cavalli - non possiamo dichiararci soddisfatti delle approssimazioni di Hochholzer (1) che è riuscito unicamente a calcolare che il rendimento di un ettaro di vite, a quell'epoca, nella regione di Agrigento, era di 2500 1. nelle buone annate. Egli ritiene d'altronde che la popolazione dell' isola era allora di 1500000 abitanti.

La fotografia ci fa dunque pensare in primo luogo a un paesaggio fiorente e a una città attiva, giacchè accanto all'agricultura anche il commercio e l'artigianato occupavano un posto importante nelle colonie greche. Il territorio di Selinunte era atraversato da un piccolo fiume le cui rive erano coperte da prezzemolo, pianta che diede nome alla località.

La città avera il grave inconveniente di essere ubicata vicino alle paludi e fu necessaria tutta l'abilità del filosofo e ingegnere Empedocle per prosciugarle. Per contro piu favorevole la posizione dell'acropoli circondato com'era da due braccia di mare, oggi insabbiate, che servivano l'uno da porto di guerra l'altro da porto commerciale. L'attività di quest'ultimo era principalmente diretta verso Cartagine. Volendolo, potremmo ora abbandonarci a romantici sogni sulla vita nella Grande Grecia. Ma non ne abbiamo affatto l'intenzione.

Queste colonne e queste pietre sono delle rovine, essendo Selinunte stata distrutta dai Cartaginesi nel 409 a.C. Ma queste rovine, ricordandoci il seguito della storia della Sicilia, sono per noi di grande interesse in quanto ci permettono di paragonare il volto antico a quello moderno suggerendoci nuovi problemi geografici ed economici, indicandoci nuove soluzioni. Dopo la caduta dell'impero romano e l'indebolimento di Bisanzio, la Sicilia fu invasa e colonizzata dai Saraceni che, nel XIIo seccolo, costituivano circa un terzo della popolazione dell'isola. Essi istituirono varie nuove colture, come il cotone, il riso e soprattutto la canna da zucchero. L'irrigazione ricevette un forte impulso, ciò che permise un rinnovamento dell'agricultura.

E' probabile che la situazione economica peggiorò in seguito all'invasione dei Normanni. In modo particolare venne a mancare l'approviggionamente d'acqua e le foreste deperirono.

Sotto la dominazione spagnola, dal 1282 al 1713, prevalse il regime della grande proprietà. Ciò provocò un abbandono sempre più accentuato della cultura dei cereali; tutti gli sforzi vennero concentrati sulla canna da zucchero. Il vigneto si sviluppa a poco a poco. L'agricultura sembra destinata a una monocultura speculativa. Purtroppo nulla sappiamo sulle condizioni del suolo, la struttura agraria e la produttività a quell' epoca. Sappiamo soltanto che la produzione locale non basta a nutrire la popolazione e che si devono importare dei cereali. Le cifre della dogana di Palermo ce lo confermano. 


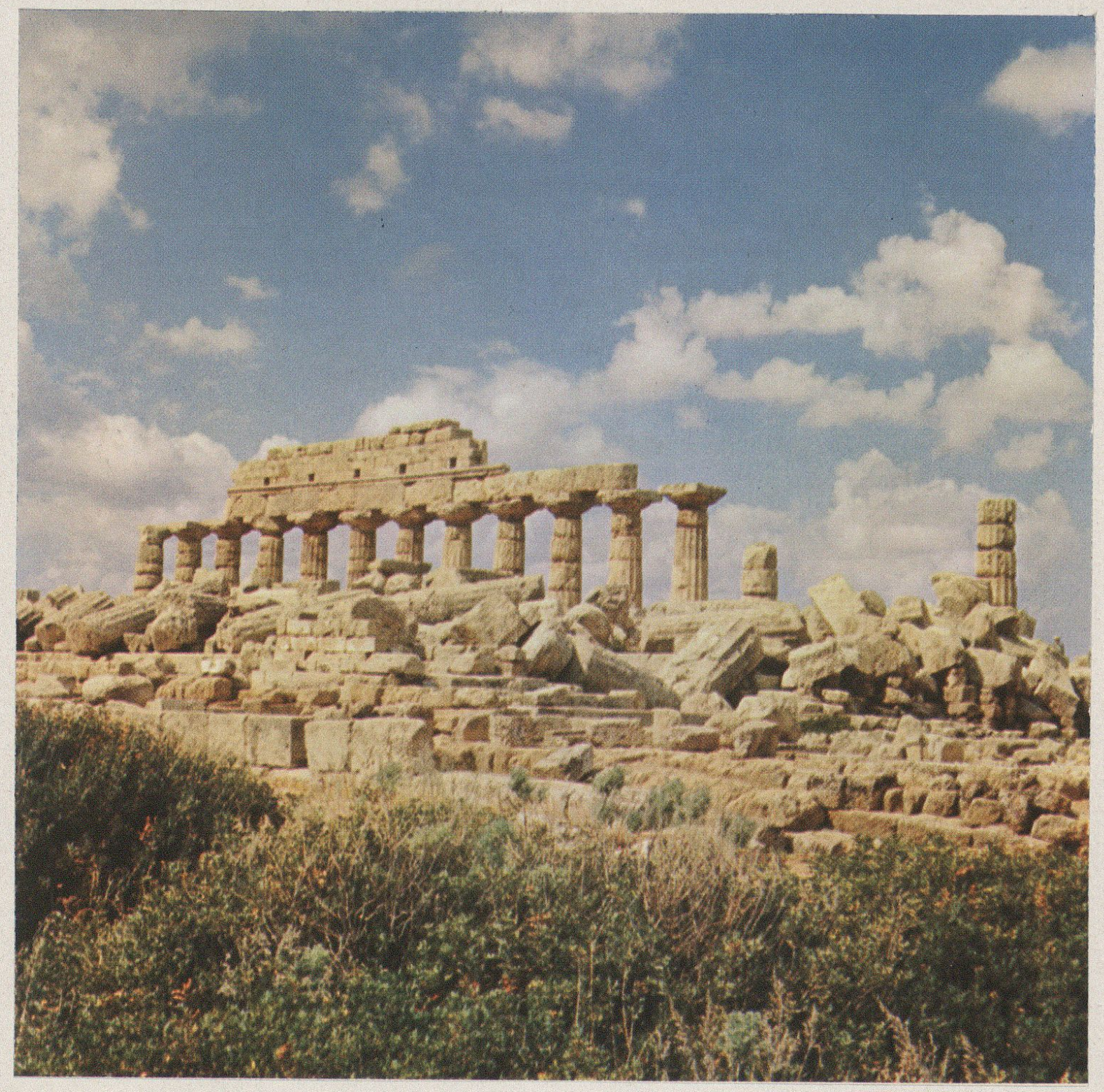

SELINUNTE

Tempio C 
Più tardi la situazione migliorò ; la canna da zucchero fu sostituita dal tabacco e lo Stato, che d'allora in poi proteggerà l'economia del paese mediante solide barriere doganali, incoraggia la cultura degli olivi.

E' in seguito all'unità italiana che l'Italia meridionale e la Sicilia verranno considerate per molto tempo come paesi sotto-sviluppati; conseguentemente vennero soppressi i primi tentativi d'industrializzazione che avevano allora una forma ancora artigianale (2).

Fino all'anno 1800 circa, la popolazione della Sicilia non aveva mai oltrepassato 1600000 abitanti. E' a questo punto che ebbe inizio il rapido e improvviso sviluppo demografico che è al giorno d'oggi uno dei problemi più angosciosi dell'economia mondiale. Nel 1951 la popolazione della Sicilia era di circa 4500000 abitanti, ma le condizioni naturali non avevano migliorato; il regime agrario si modificava solo molto lentamente e la seconda guerra mondiale aveva devastato quest'isola già cosí scarsamente munita di mezzi di comunicazione e di servizi centrali di ogni genere. Se non possiamo parlare in modo negativo dell'economia di tutte le regioni della Sicilia - giacchè le condizioni naturali e umane variano e certe regioni sono produttive e forniscono degli alti rendimenti - possiamo tuttavia dire che questa fotografia delle rovine di Selinunte ci rivela indirettamente un drammatico probleme, quello di un'isola troppo popolata i cui abitanti non possono più avere un livello di vita decente.

E'vero che nel 1950 fu creata la Cassa per il Mezzogiorno, impresa officiosa incaricata di migliorare le condizioni di vita dell'Italia meridionale, compresa la Sicilia, nell' ambito di un piano di 12 anni. In 5 anni questa organizzazione ha già speso 108 miliardi di lire per la Sicilia (bonifica di terre, irrigazione, costruzione di strade), ma solo un' intensa industrializzazione o un'importante emigrazione potrebbero diminuire la pressione demografica (3). Purtroppo la Sicilia non manca solo di fabbriche, capitali e operai qualificati, ma anche di materie prime. La sola industria che sembra destinata a svilupparsi è il turismo.

Date queste circostanze, è più facile capire lo sviluppo del banditismo, l'aumento della disoccupazione e la sotto-alimentazione di un proletariato agriccolo estremamente povero. I tentativi di DANILO DoLcI, benchè limitati, hanno sottolineato la necessità di far partecipare attivamente la popolazione della Sicilia agli sforzi fatti per dare un nuovo slancio all'economia dell'isola, dalla quale dipende il livello di vita dei suoi abitanti (4). Questa è una condizione indispensabile per far riuscire qualsiasi piano per il miglioramento del territorio.

Speriamo dunque che il turismo, che è una fonte non trascurabile di reddito per l'isola, continui a contribuire al suo sviluppo economico. Anche Danilo Dolci si recò in Sicilia la prima volta per ammirare le rovine dell'antichità!

\section{B I B LIOG R A F I A}

(1) Hochнolzer, H.: Historische Kulturgeographie des großgriechischen Sizilien. Klio, Beiträge zur alten Geschichte 1935, p. 92. - (2) BIROT. P. et Dresch, J.: La Méditerrannée et le Moyen Orient. Paris 1953, T.I.p. 384-385. - (3) Compiti e realizzazioni della Cassa per il Mezzogiorno. Estratto dal volume La Cassa per il Mezziogiorno-Prima quinquennio: 1950-1955, Roma 1956, p. 19. - (4) DolcI, D.: Inchiesta a Palermo. S. 1. 1956.

Ringraziamo la casa editrice Kümmerly \& Frey per la gentile concessione delle fotografie a colori pubblicate nel libro «Sonneninsel Sizilien» di F. JUNKER.

\section{SELINUNT, EINE HISTORISCHE LANDSCHAFT SIZILIENS}

Die Ende des 8. Jahrhunderts v. Chr. gegründete Stadt Selinunt war bis zu ihrer Zerstörung 409 v. Chr. eine reiche und betriebsame Siedlung des griechischen Sizilien. Seine Ruinen sind ein sprechender Hinweis auf die zahlreichen und schwierigen Meliorationsprobleme einer einst prosperierenden, heute überbevölkerten Insel. 